\title{
Bubbles navigating through networks of microchannels
}

\section{Citation}

Choi, Wonjae, Michinao Hashimoto, Audrey K. Ellerbee, Xin Chen, Kyle J. M. Bishop, Piotr Garstecki, Howard A. Stone, and George M. Whitesides. 2011. "Bubbles Navigating through Networks of Microchannels." Lab on a Chip 11, no. 23: 3970-3978.

\section{Published Version}

doi:10.1039/c1lc20444k

\section{Permanent link}

http://nrs.harvard.edu/urn-3:HUL.InstRepos:12876666

\section{Terms of Use}

This article was downloaded from Harvard University's DASH repository, and is made available under the terms and conditions applicable to Open Access Policy Articles, as set forth at http:// nrs.harvard.edu/urn-3:HUL.InstRepos:dash.current.terms-of-use\#OAP

\section{Share Your Story}

The Harvard community has made this article openly available.

Please share how this access benefits you. Submit a story.

\section{Accessibility}




\section{Bubbles Navigating through Networks of Microchannels}

Wonjae Choi, Michinao Hashimoto, Audrey K. Ellerbee, Xin Chen, Kyle J. M. Bishop, Piotr

Garstecki ${ }^{\dagger}$, Howard A. Stone ${ }^{\ddagger}$, and George M. Whitesides ${ }^{*}$

Department of Chemistry and Chemical Biology, Harvard University,

12 Oxford Street, Cambridge, MA 02138

† Institute of Physical Chemistry, Polish Academy of Sciences, Kasprzaka 44/52, 01-224

Warszawa, Poland

$\$$ Department of Mechanical and Aerospace Engineering, Princeton University, Princeton, NJ 08544

*Corresponding Author

Email Address: gwhitesides@gmwgroup.harvard.edu 


\section{Abstract}

This paper describes the behavior of bubbles suspended in a carrier liquid and moving within microfluidic networks of different connectivities. A single-phase continuum fluid, when flowing in a network of channels, partitions itself among all possible paths connecting the inlet and outlet. The fluid determines its relative flow rates along different paths by interacting with the global structure of the network. That is, the distribution of flows depends on the fluidic resistances of all channels of the network. The movement of bubbles of gas, or droplets of liquid, suspended in a liquid can be quite different from the movement of a single-phase liquid, especially when they have sizes slightly larger than the channels, so that the bubbles (or droplets) contribute to the fluidic resistance of a channel when they are transiting it. This paper examines bubbles in this size range; in the size range examined, the bubbles are discrete and do not divide at junctions. As a consequence, a single bubble traverses only one of the possible paths through the network, and makes a sequence of binary choices ("left" or "right") at each branching intersection it encounters. We designed networks so that, at each junction, a bubble chooses the channel into which the volumetric flow rate of the carrier liquid is highest. When there is only a single bubble inside a network at a time, the path taken by the bubble is, counter-intuitively, not necessarily the shortest or the fastest connecting the inlet and outlet. When a small number of bubbles move simultaneously through a network, they interact with one another by modifying fluidic resistances and flows in a time dependent manner; such groups of bubbles show very complex behaviors. When a large number of bubbles (sufficiently large that the volume of the bubbles occupies a significant fraction of the volume of the network) flow simultaneously through a network, however, the collective behavior of bubbles - the fluxes of bubbles through different paths of the network - can resemble the distribution of flows of a single-phase fluid. 


\section{Introduction}

Networks - which can be represented abstractly by sets of nodes, joined by edges - are ubiquitous. ${ }^{1-3}$ When constructed by humans, one of the major functions of networks is to transport (or describe the transportation of) components - e.g., people and freight (highways and rail systems), information (the internet, chemical networks), cargo (logistics systems), water (urban water systems), and energy (food webs and power grids). In some of these networks (e.g., power grids), the components (electrons) are passive and driven by external sources (electrical potential); in others (e.g., highways), the components (vehicles) can be powered and active. Both are important. The objective of the work described in this paper was to understand the mechanisms by which passive components select their paths from the inlet to outlet through a model network. We believe the understandings from this work will be helpful in the design of synthetic networks through which discrete components move in ways that solve problems in analysis, and in computation and simulation.

We describe systems comprising bubbles traveling through microfluidic networks. In these systems, we chose the sizes of the bubbles explicitly to be such that the bubbles interact with the walls of the channels. We used these systems to study the principles that dictate the path followed by the bubbles - passive, but mutually interacting components - and especially their choice of paths at junctions. The junctions and channels in the fluidic networks studied here correspond to nodes and edges in an abstract network. To simplify the problem, we focused on networks based on T-junctions, in which the bubbles have only binary choices ("left" or "right").

For a mathematical network comprising only nodes and edges, it is straightforward for a computer to find a path that optimizes a particular parameter (e.g., the shortest path from inlet to outlet, or the path with the smallest number of nodes), by systematically trying all paths and 
building a look-up table. ${ }^{4-5}$ The time required for these computations is roughly proportional to the square of the number of nodes, except in networks with sparse connections; ${ }^{4}$ thus the process becomes extremely time-consuming for highly complex networks. More importantly, there is no practical process for calculating paths through real networks - that is, networks with non-linear properties, with interactions between components, with resistances to motion that depend on the details (geometry, flow rates, etc) in complex ways, and especially, with dynamic characters, i.e., properties that change with time.

For most real networks, the components do not have a complete map of the global structure of the networks. For example, individual erythrocytes in a circulatory system do not follow a globally planned path, and drivers of cars navigating traffic respond to its dynamic character, but their decisions are based on incomplete information. In such cases, the components interact primarily with the portion of the network in their immediate proximity (the "local" portion), although they may also interact more weakly with more distant (non-local) parts of the system. They thus choose their paths based principally on local information (although the local environment may, of course, be strongly influenced by distant structures, or by the dynamics of other components). In addition, the local interactions governing the behavior of components of the system may evolve both in space and time; dynamic evolution makes the modeling of these kinds of network problems difficult or impossible.

Attempts to solve problems involving real networks have motivated studies of analog systems probing primitive networks of channels (for example, glow-discharge arcs, flows of liquids, and growing amoeboid colonies) to give "shortest path" solutions to mazes. These studies have been used to suggest solutions to problems according to various criteria. ${ }^{6-11}$ One major difference between the systems used in these experimental studies and computer algorithms is the fact that the analog systems find optimal pathways by comparing the 
characteristics of all paths in parallel, while the computer builds its look up table serially. Also, since the physical systems are dissipative, they show non-linear and dynamic characteristics. Therefore, in theory, these analog systems might have advantages over computer systems in solving complex, time-varying mazes, and related types of problems. In practice, in the systems described in the literature to date, ${ }^{6-11}$ the results have not been sufficiently generic or generalizable to be useful: the behaviors of these model systems are based on the interaction between continuums (e.g., fluids or plasmodia) and the entirety of simple networks, while many real networks comprise discrete components (e.g., erythrocytes) in fluids and channels with extended, complex structures. The results from the studies of simple continuous systems cannot be extended with any assurance to more complex, heterogeneous systems with discrete components.

As a model system with which to explore network problems incorporating discrete components, this paper uses a system comprising three elements: i) a network of interconnected microchannels, ii) a carrier fluid (water) flowing at low Reynolds number, driven by a pressure difference between the inlet and outlet of the network, and iii) bubbles of nitrogen suspended in the fluid. Critically, in this study we selected the diameter of the bubbles to be slightly larger than the cross-section of the channel. The surfaces of the bubbles thus interact with the walls of the channels, and alter the pressure drops and flow rates along the channels these bubbles occupy; as the bubbles move among channels, the resulting variations in flows propagate across the network at the speed of sound in water, and allow all the bubbles to mutually interact. These systems of moving, interacting bubbles lead to dynamic behaviors that are much more complex than those in simple, single-component microfluidic systems. 
The dynamics of a bubble or a droplet moving in a single channel has been studied for decades. ${ }^{12-14}$ The understandings from these studies make it possible to rationalize the behavior of bubbles moving in networks of minimal complexity (e.g., two parallel channels with constant cross-sectional area, and a common inlet and outlet). ${ }^{15}$ The dynamics in those relatively simple, two-path networks still generate a wide range of complex patterns of flows, including bistability, filtering, memory effects and bifurcations from a regular periodic movement to irregular or regular with a very long period. ${ }^{15-20}$ Flow patterns of bubbles and droplets in networks that have more complicated connectivity have also been reported. ${ }^{21-23}$

Flow of a Single-Phase Fluid. Figure 1a is a schematic showing a volume of a singlephase fluid (light gray) being injected into a network that is already filled with the same type of fluid (dark gray; the two volumes of liquid are shaded differently only for better visualization). At an intersection, this fluid splits and merges, and thus partitions between the two paths leading from inlet to outlet (Fig. 1a; $Q_{A}, Q_{B}>0, Q$ represents the volumetric flow rate). The incompressible, pressure-driven flow of a single-phase Newtonian fluid at low Reynolds number has a velocity $u(x, y, z)$ at any position that satisfies the linearized Navier-Stokes equation (Eq. 1) with no-slip boundary condition (Eq. 2): ${ }^{24}$ here $\mu$ is the dynamic viscosity of the fluid $\left(\mathrm{kg} \mathrm{m}^{-1} \mathrm{~s}^{-1}\right)$, and $p$ is the pressure $\left(\mathrm{kg} \mathrm{m}^{-1} \mathrm{~s}^{-2}\right)$.

$$
\begin{aligned}
& \mu \nabla^{2} u-\nabla p=0 \\
& u=0, \text { at the solid surfaces }
\end{aligned}
$$

Equations 1 and 2 describe a time-invariant flow field in the entire network. Importantly, the behavior of a single-phase fluid in any region (for example, the partitioning of flows at the inlet junction in Fig. 1a, and the resulting flow rates $Q_{A}$ and $Q_{B}$; hereafter, the flow rate means 
the volumetric flow rate and has a unit of $\mathrm{m}^{3} \mathrm{~s}^{-1}$ ) is determined by the structure of the entire network (that is, the "global" structure), not simply by the structure of the closest junction. The solution of Eq. 1 minimizes a parameter - the rate of viscous energy dissipation for the total flux through the given geometry (Eq. 2), in the case of zero Reynolds number. ${ }^{24-25}$

It is difficult to obtain an analytical solution for velocity field for a single-phase fluid flowing through an arbitrarily shaped geometry. It is possible, however, to estimate flows in a network that comprises straight channels and junctions: for a flow in a long, straight channel with a rectangular cross-section, solving Eqs. 1 and 2 predicts that the relation between the pressure drop and the flow rate through the channel can be approximated by Eq.3. In Eq. 3, $Q$ is the volumetric flow rate $\left(\mathrm{m}^{3} \mathrm{~s}^{-1}\right), \Delta P$ is the drop of pressure across the length of the channel $(\mathrm{kg}$ $\mathrm{m}^{-1} \mathrm{~s}^{-2}$ ), and $w, h, L$ are the width, height, and length of the channel (m; Eq. 3 is valid as long as $w \geq h$; in the current work we designed all channels so that $w=100 \mu \mathrm{m}, h=15 \mu \mathrm{m}){ }^{26}$

$$
\Delta P=P_{\text {upstream }}-P_{\text {downstream }} \approx Q \frac{3 \mu L}{4 w h^{3}}\left[1-\frac{192}{\pi^{5}}\left(\frac{h}{w}\right)\right]^{-1}
$$

Equation 3 can be re-written into Eq. 4 , which is an analog of Ohm's law, $\Delta V=I R_{\text {elec }}$, for an electrical circuit: the drop in pressure $\Delta P$ replaces the drop in voltage $(\Delta V)$ across two points in the circuit, and the flow of liquid $Q$ replaces the flow of electrons (electrical current, $I) .{ }^{27}$ It is then possible to define a fluidic resistance $R\left(\mathrm{~kg} \mathrm{~m}^{-4} \mathrm{~s}^{-1}\right.$; see Eq. 5) for a single channel - a parameter analogous to electrical resistance $\left(R_{\text {elec }}\right)$ - based on this analogy.

$$
\begin{aligned}
& \Delta P=Q R \\
& R=\frac{3 \mu L}{4 w h^{3}}\left[1-\frac{192}{\pi^{5}}\left(\frac{h}{w}\right)\right]^{-1}
\end{aligned}
$$

The concept of fluidic resistance makes it straightforward to determine the rates of flow of a single-phase fluid through all channels of any complex network, by combining the 
resistances of all channels composing the network. The procedure is similar to those used with electric circuitry: two resistances connected in parallel or in series combine such as $R_{\text {parallel }}=\left(1 / R_{1}+1 / R_{2}\right)^{-1}$ and $R_{\text {series }}=R_{1}+R_{2}$. Networks with more complicated connectivities require fluidic analogs of Kirchhoff's rules. Thus, once the distribution of the fluidic resistances of all channels (and their connectivity) is known, the flow rates in all channels can be readily calculated. Of course, channels in microfluidic networks can have features (e.g., changes in the cross-sectional area, angled connections, corners, and so forth) that might make the fluidic resistances of the channels deviate from the simple estimates given by Eq. 3. We maintained a constant cross-sectional area for all channels to minimize the effects of such irregularities.

The analogy to Ohm's law suggests that, for a single-phase fluid, the flow rates dividing and merging at any junction of a network are determined by contributions from fluidic resistances along all channels connecting the various junctions or intersections, not simply by the geometry of that junction. This conclusion is not a surprise, considering the fact that the analogy to Ohm's law is based on the assumption that the flow is in the Stokes regime (Eq. 1), in which the entire flow field is explicitly determined by the global structure of the network (Eq. 2).

Figure $1 \mathrm{a}$ and $1 \mathrm{~b}$ show two demonstrations of a single-phase flow based on the distribution of fluidic resistances across the entire network, not just on the geometry of a particular T-junction. For a two-path network possessing different fluidic resistances along its two paths (Fig. 1a), despite the clear symmetry of the inlet junction, the flow rate would be higher along the path whose fluidic resistance is lower than the other $\left(Q_{A}>Q_{B}\right)$. Figure $1 \mathrm{~b}$ shows the opposite case: the network possesses two channels that are symmetric to each other with respect to point $\mathrm{C}$ in the center. For such a point-symmetric geometry, the velocity field of a Stokes flow (i.e., zero Reynolds number) should be point-symmetric as well (with the obvious 
exception of the orientation of flow). The volumetric flow rates in two channels should thus be the same, meaning that the fluid incoming into the inlet junction partitions equally into the two downstream channels $\left(Q_{A}=Q_{B}\right)$, despite the obvious asymmetry of the inlet junction. One can verify this hypothesis using a numerical simulation (see supporting Fig. S1 for a velocity field of a hypothetical flow possessing zero Reynolds number in a point-symmetric two-path network, simulated using COMSOL software).

Selection of Path by a Single Bubble. In contrast to a continuum fluid, a bubble is a discrete entity that follows only one of all possible paths (unless it splits; bubbles do not split in the experiments described in this work). ${ }^{26}$ Figure $1 \mathrm{c}$ shows a schematic of a bubble at a T-shaped junction, in which a bubble selects the channel to follow by making a binary decision to proceed left or right at the junction. A bubble makes such binary decisions by interacting with the carrier liquid around it. Obtaining an analytical prediction for the movement of a bubble is, in general, extremely difficult. ${ }^{28}$ For a junction of the shape shown in Figure 1c (symmetric T-junction, in which the upstream channel makes an orthogonal connection with two downstream channels), however, it is possible to simplify the problem and predict the channel a bubble will choose: when a bubble is large enough to fill the entire junction shown in Fig. 1c, due to the orthogonal connection between the upstream and downstream channels, the interaction between the bubble and the carrier liquid in the upstream region (occurring at the liquid-gas interface; dotted line in Fig. 1c) does not contribute to the path-selection of a bubble. A bubble thus selects the channel to follow by interacting with the carrier liquid flowing into the two downstream channels (dashed and dash-dotted lines in Fig. 1c). At the moment a bubble reaches a junction, the pressures in the two downstream channels are essentially the same in the proximity of the junction. In addition, the symmetric geometry of the junction ensures that the effects from the interfacial tensions in 
the two downstream channels will also be the same and cancel each other. Hence the movement of a bubble will be determined by the difference between the velocities of the carrier fluid (integrated over the liquid-gas interfaces) along the two downstream channels. These two integrals, when the two channels have the same cross-sectional area, are simply proportional to the flow rates into the channels. In other words, when a bubble reaches a T-junction, for which two downstream channels have the same cross-sectional area, the bubble chooses the channel that possesses the higher flow rate $\left(\mathrm{m}^{3} \mathrm{~s}^{-1}\right)$.

Figure 1d shows the path-selection of a bubble in a two-path network, whose anisotropy is similar to the one in Fig. 1a. Both channels in the network have the same cross-sectional area; hence, it is obvious from Eq. 5 that the fluidic resistance of shorter channel (channel A) is lower than that of channel B. The flow rate is thus higher along channel A than along B, and a single, isolated bubble travels through channel A. The path-selection by a bubble is not, however, simply determined by the flow rates if the cross-sectional areas of the two downstream channels are different. Figure 1e shows a network possessing point-symmetry (e.g., Fig. 1b), where the volumetric flow rates $\left(\mathrm{m}^{3} \mathrm{~s}^{-1}\right)$ along two channels are the same. Figure 1e illustrates the fact that, despite the symmetry of the network and the equal flow rates along two channels, bubbles consistently choose channel B. We suspect the observed path-selection was due to the difference in velocities of the carrier fluid into two channels. Further analysis of the path-selection of bubbles at asymmetric junctions such as the one in Fig. 1e will be the topic of a subsequent paper. For the rest of the current paper, we used only the T-junctions of which the downstream channels have an equal cross-sectional area so that the flow rate and the velocity of the carrier liquid are explicitly proportional to each other.

In summary, given a fluidic network comprising microchannels and T-junctions of symmetric geometry, a single-phase fluid continuously allocates itself among all channels by simultaneously interacting with the global structure of the entire network, that is, the distribution 
of fluidic resistances of all channels and their connectivity through the entire network. Such an interaction leads to a stationary Stokes flow through the network. In the same network, a bubble makes a sequence of binary choices at each junction by interacting with the carrier fluid at that local junction, that is, the flow rates $\left(\mathrm{m}^{3} \mathrm{~s}^{-1}\right)$ along channels connected to that junction. Due to these two characteristics of the path-selection by a bubble - selection is binary, and determined based on the local information - a system of bubbles is a convenient and tractable tool for us to explore the navigation of discrete components through complex networks. In the rest of this paper, we report the flows of bubbles in modestly complicated networks, with different topographies and connectivities, and with different numbers of bubbles moving in the networks.

We acknowledge that simple systems of bubbles moving in microchannels will not model all types of behavior in real, complex networks (for example, active lane changing of vehicles an extreme instance in which components are under active control and respond to both shortrange, visual information and long-range information offered by GPS). Nevertheless, the simple systems that we study here possess the archetypal ingredients leading to the complexity of movement of discrete components in networks: each component (bubble) travels through the network by making binary choices at junctions based on the local information (the relation between flow rates at that junction), and that local information is affected by the presence of other components (that is, the flow rates are modified by other bubbles). We anticipate that understanding such elementary systems will help to understand more complex ones, and will also provide experimental data useful in testing theoretical models and simulations. 


\section{Results and Discussion}

The Path-Selection of a Single Bubble in Complex Networks. Figure 2 shows a network that has three paths leading from the inlet to the outlet (Fig. $2 \mathrm{a} ; \mathrm{A}, \mathrm{B} \rightarrow \mathrm{C} \rightarrow \mathrm{E}$, and $\mathrm{B} \rightarrow \mathrm{D} \rightarrow \mathrm{E})$. We fixed the cross-sectional area of all channels in the network so that the fluidic resistances given by Eq. 5 are approximately, neglecting the influence of corners, proportional to the lengths of the channels. Calculations based on the distribution of fluidic resistances predict that the relative flow rates in all channels should be $Q_{A}: Q_{B}: Q_{C}: Q_{D}: Q_{E}=$

\section{$1: 1.13: 0.60: 0.53: 1.13\left(Q_{B}\right.$ and $Q_{E}$ are simply $\left.Q_{B}=Q_{E}=Q_{C}+Q_{D}\right)$. The estimated ratio} between the flow rates along different channels can be readily verified by a numerical simulation (Fig. 2b, $Q_{A}: Q_{C}: Q_{D}=1: 0.63: 0.55$ ) or by particle image velocimetry (Fig. 2c, $\left.Q_{A}: Q_{C}: Q_{D}=1: 0.60: 0.50\right)$. Based on these ratios, it is possible to estimate the residence time of a passive tracer flowing with the locally superficial speed along the three paths. If a tracer chooses path $\mathrm{B} \rightarrow \mathrm{C} \rightarrow \mathrm{E}$ or $\mathrm{B} \rightarrow \mathrm{D} \rightarrow \mathrm{E}$, it will pass through channel $\mathrm{C}$ or $\mathrm{D}$, in each of which the volumetric flow rate of the carrier fluid is lower than that through path A. As a result, the relative residence times along the three paths $\left(\tau_{A}: \tau_{B C E}: \tau_{B D E}\right)$ are $1: 1.50: 1.78$, suggesting that path $\mathrm{A}$ is the shortest (by length), fastest (by time) path for a bubble to travel through the network. Such measures that are associated with paths connecting the inlet and outlet are, however, irrelevant in the path-selection of a bubble: Figure $2 \mathrm{~d}$ is a composite of seven successive still images of a single bubble traversing the fluidic network used in Fig. 2a (The Experimental section describes the details of the bubble generation). The bubble took path $\mathrm{B} \rightarrow \mathrm{C} \rightarrow \mathrm{E}$ (i.e., the path of intermediate length) rather than path A (i.e., the shortest path, which also allows the bubble the briefest exit from the outlet). 
We can explain the path-selection of the bubble shown in Fig. $2 \mathrm{~d}$ based on the fact that this bubble chooses the channel to enter at each junction using the relation between the flow rates into the channels connected to that junction (responding to local flow rates at the proximity of the bubble); path-selection is not determined by the flow rates along the paths connecting the inlet and outlet of the network. A bubble at the inlet junction chooses one of two channels to enter simply by following the more rapid flow - e.g., $Q_{B}$ rather than $Q_{A}$. The parallel connection between channels $\mathrm{C}$ and $\mathrm{D}$ makes the flow rate in channel $\mathrm{B}$ higher than that in $\mathrm{A}$ $\left(Q_{B}=\left(Q_{C}+Q_{D}\right)>Q_{A}\right)$, and results in the bubble - when at the first T-junction - choosing channel $\mathrm{B}$ rather than channel $\mathrm{A}$. When the bubble reaches the second intersection, it again chooses between two channels (channels C and D). In both, there is the same pressure drop across the length of the channel, but channel $\mathrm{C}$ is the shorter, and thus possesses a lower fluidic resistance than $\mathrm{D}$. The flow rate of the carrier fluid is therefore higher along channel C than $\mathrm{D}$, and the bubble selects channel C rather than D. It finally exits through E to the outlet.

Figure 3 provides a more striking comparison between the path-selections based on global and local information. For this highly anisotropic, multi-path network, calculations using the global distribution of fluidic resistances predict that the volumetric flow rate of a singlephase fluid through path A should be $44 \%$ of the total, while the flow rate through path B (the flow rate along path $\mathrm{B}$ varies along the length of the path, and we measured the lowest value at the channel indicated with an arrow) would be only $4.6 \%$. Despite this $\times 10$ difference in the flow rates, isolated bubbles travel exclusively through path $\mathrm{B}$. The bubble travels into the longer, slower path because, at each junction, the flow rate into the channel comprising path $\mathrm{B}$ is higher than the other due to the parallel connectivity of the channels in that portion of the network. There is no further bifurcation after junction $\mathrm{T}$; hence, the flow rate and the length of each 
channel exiting junction $\mathrm{T}$ are inversely proportional. Therefore, uniquely at the junction $\mathrm{T}$, the bubble chooses the shorter channel.

Calculations based on the flow rates and lengths of the channels estimate that, ignoring the effect of the bubble on the flow of the carrier fluid, the residence time of a bubble traveling along the observed path (path B) is 7.7 times longer than the time in a hypothetical case that a bubble chooses path A. In other words, a bubble makes a sequence of choices at each junction, each of which it moves into the channel with the highest flow rate (or the highest velocity of the carrier fluid, since the cross-sectional area in all channels is the same). This sequence of choices, however, leads it into the second-longest path from the inlet to outlet of the network. The examples in Figs. 1 to 3 demonstrate that the navigation of a bubble is simply a sequence of binary choices that does not necessarily solve the given maze to yield solutions such as the ones allowing the shortest length or the shortest time for the bubble to pass through the network.

Mutual Interactions Among Multiple Bubbles. An important characteristic of these systems of bubbles - bubbles with sizes comparable to that of a channel - is that each bubble increases the fluidic resistance of the channel through which it moves. ${ }^{15}$ The presence of a bubble in one channel then changes the flow rates of the carrier fluid in all of channels in that network; this ability of a bubble to modulate the distribution of flow rates in the entire network provides a mechanism for communication (at the speed of the propagation of pressure; e.g., the speed of sound in the liquid) among all bubbles in the network. (For the networks described here, and for a speed of sound of $1500 \mathrm{~m} / \mathrm{s}$ for water, the transit time for information between the inlet and outlet is therefore $\sim 1 \mu \mathrm{s})$. The system of bubbles in networks of microfluidic channels thus 
emerges, we believe, as a tractable one for studying the characteristics of complex, dissipative systems.

The fluidic resistance associated with a bubble is dependent on the surface tension of the liquid-gas interface, the velocity and size of the bubble, and the dimensions of the channel. ${ }^{29}$ The velocity of the bubbles in all channels of the network shown in Fig. 2 are on the same order (see Fig. 2C, in which the velocities of the tracers in channel A, C, and D were 16.6, 9.93, and 8.36 $\mathrm{mm} / \mathrm{s}$ ) and the size of the bubbles was sufficiently uniform (polydispersity $\sim 1.04)^{30}$ that the fluidic resistance added by the bubbles can be approximated to be almost a constant. ${ }^{29,31}$ In such a case, Equation 6 estimates the total resistance associated with a channel containing bubbles, where $n$ and $R_{B}$ are the number of bubbles in the channel and the resistance that a single bubble adds to the channel. (A full description on the origin of this added resistance is not the focus of the current paper, and we refer readers interested in further details to our previous work ${ }^{29}$ or the work of Wong et al. ${ }^{13}$ or Hodges et al. ${ }^{12}$ ) Since the number of bubbles in a particular channel changes with time as bubbles enter and exit, the fluidic resistance of each channel also varies with time. The electrical analog of this microfluidic system would be an electrical circuit in which resistors move through the circuit.

$$
R_{\text {eff ective }}(t)=R+n(t) R_{B}
$$

Figure 4 demonstrates two examples of such mutual interactions. Here we used the network shown in Fig. 2a and increased the number of bubbles in the network (by decreasing the flow rate of water to $2.5 \mathrm{~mL} / \mathrm{hr}$ while maintaining the inlet pressure of nitrogen at $34 \mathrm{kPa}$ ). In the example illustrated in Figs. 4a-e, as bubble $\alpha$ moves through channel E, it increases the fluidic resistance of this channel, and thereby decreases the flow rate of liquid through both paths $\mathrm{B} \rightarrow \mathrm{C} \rightarrow \mathrm{E}$ and $\mathrm{B} \rightarrow \mathrm{D} \rightarrow \mathrm{E}$ (i.e., $\left.Q_{B}=Q_{E}=\left(Q_{C}+Q_{D}\right)\right)$. Thus the flow rate through channel A 
becomes higher than through $\mathrm{B}$, and a second bubble $(\beta)$ entering the system chooses channel $\mathrm{A}$. The subsequent bubble $(\gamma)$ follows $\mathrm{B} \rightarrow \mathrm{C} \rightarrow \mathrm{E}$, since at this rate of entry of bubbles into the network, this path is again free of bubbles.

Reducing the flow rate of water by $<0.5 \%$ (from $2.50 \mathrm{~mL} / \mathrm{hr}$ to $2.49 \mathrm{~mL} / \mathrm{hr}$ ) increases the number density of bubbles, and leads to a very different pattern of navigation of bubbles: in this case, $\beta$ reaches the first intersection while $\alpha$ is still traveling through channel $\mathrm{C}$, which thus has a higher resistance than in the absence of $\alpha$ (Figs. $4 \mathrm{f}-\mathrm{j}$ ). The overall influence of bubble $\alpha$ on the flow rate (and the gradient of pressure) along channel B is weaker than the previous case (Figs. 4a-e), because $\alpha$ increases the resistance of only one (C) of the two parallel channels C and D. Therefore, $\beta$ travels through $\mathrm{B}$ after passing the first intersection, and along $\mathrm{C}$ after passing the second intersection, because $\alpha$ has already exited the channel $\mathrm{C}$ by then. When $\gamma$ enters the network, it follows the path taken by $\beta$. A comparison between the patterns of flows shown in Figs. 4a-h reveals that the spatial distribution of bubbles in a given network at the very moment when a trailing bubble occupies each junction determines the selection of its path. Table S1 summarizes the detailed correlation between the occupancy of channels and the selection of paths by bubbles entering the network.

\section{The Navigation of Multiple Bubbles in Complex Networks - Propagation of}

Information. The mutual interaction between bubbles can be considered as a mechanism by which bubbles at various locations in a network receive and transmit information both upstream and downstream about the geometric structure of the network, and thereby influence the decisions of both leading and following bubbles. The first bubbles entering the network do not choose the path to follow based on the average measure of the path (e.g., the distance to travel or 
the residence time); they simply follow a sequence of channels each of which possesses the highest flow rate at each branching intersection. Having entered a channel, each bubble increases the fluidic resistance of that channel; this increase correspondingly lowers the flow rate leading into that channel, and also affects the flow rates along all other channels. This change in the flow rate in one channel, thus, influences the choice of channels at all junctions; in particular, it reduces the tendency of the next bubble to follow the same path as that of a bubble already occupying a particular channel. As soon as the bubble leaves the channel, that channel returns to its original fluidic resistance. We thus infer that, as the number density of bubbles increases, the diversity of paths taken by new bubbles increases such that new bubbles populate more and more of the channels connecting the inlet and outlet.

Figure 5 summarizes an experiment in which we varied the number density of bubbles in the channels, and observed the selection of paths by these bubbles. By observing the pathselections of bubbles when the network has different distributions of bubbles (see Table S1 for further details), we estimate empirically that the resistance of each bubble in the test setup we used (velocity and length of a bubble were $\sim 10 \mathrm{~mm} / \mathrm{s}$ and $100 \sim 150 \mu \mathrm{m}$ ) is equivalent to that of a microchannel of $0.4 \sim 0.6 \mathrm{~mm}$ in length. Therefore, the additional resistance due to even a small number of bubbles can significantly affect the global distribution of resistances of the channels (the total length of all channels $=7.1 \mathrm{~mm}$ ). Consequently the mutual interaction among multiple bubbles leads to a complex, time-dependent pattern of flow (Fig. 5a). By counting the number of bubbles that pass through channels $\mathrm{A}, \mathrm{C}$, and $\mathrm{D}$, we ranked paths according to the flux of bubbles that each path can support: $\mathrm{B} \rightarrow \mathrm{C} \rightarrow \mathrm{E}$ supports the highest flux of bubbles (22), followed by $\mathrm{B} \rightarrow \mathrm{D} \rightarrow \mathrm{E}$ (13) and then by A (10). Clearly, the collective preference of multiple, mutually interacting bubbles can be very different from that of a single bubble. 
At a higher densities of bubbles ( $\sim 10$ bubbles in the network; see Fig. $5 b)$, the fluidic resistance added by bubbles is comparable to the resistance of any path connecting the inlet and outlet - path $\mathrm{A}, \mathrm{B} \rightarrow \mathrm{C} \rightarrow \mathrm{E}$, and $\mathrm{B} \rightarrow \mathrm{D} \rightarrow \mathrm{E}$. As before, each bubble takes a path through the network based only on the relation between flow rates along the microchannels at each junction it reaches, but those flow rates are heavily influenced by the many other bubbles interacting with the same network at various other locations. The ratio between the fluxes of bubbles in the three paths (22: 14: 10 in paths $\mathrm{A}, \mathrm{B} \rightarrow \mathrm{C} \rightarrow \mathrm{E}$, and $\mathrm{B} \rightarrow \mathrm{D} \rightarrow \mathrm{E}$ ) results from the ability of the system of bubbles to interact with distant parts of the network, and to share the information about the network through changing flow rates and differences in pressure. At higher values of number density, the behavior of the system does not noticeably change; instead, the ratios between fluxes converge to values (1: $0.87: 0.63)$ that approach those of the flow rates of the carrier fluid (1: 0.60: 0.53 ) when there are no bubbles. Figure $5 \mathrm{c}$ shows the collective behavior of multiple bubbles at different bubble densities. The result suggests that, as the number of bubbles increases, the system of multiple bubbles eventually begins to resemble a continuous fluid: although each of the bubbles still makes a sequence of binary choices based on the flow rates at local junctions, as a group, the bubbles partition their fluxes based on the global distribution of fluidic resistances of all channels. We plotted Figure 5c only up to the bubble density of 20; when the number of bubbles in the network increased beyond 20, a new bubble reached the first junction before its predecessor selected the channel to follow; this behavior led to direct collisions, with attendant complexities that are beyond the scope of this paper. 


\section{Conclusions}

Continuous, single-phase fluids partition flows in a network according to the structure of the entire network (i.e., the distribution of the fluidic resistances of all channels, and their connectivities). Multiple studies have used the ability of continuous fluids or other continuum phases to interact with the entirety of a network to develop analog systems that can solve problems involving physical networks. ${ }^{6-11}$ These studies, however, do not address most problems of real networks, which involve the movement of discrete components (e.g., erythrocytes or cars) which interact only with a portion of the network as they travel.

We have investigated the mechanism by which discrete components select paths to follow in moderately complicated networks. For this goal, we used a model system comprising i) networks of microfluidic channels connected through T-junctions, ii) a carrier fluid flowing through the networks, and iii) bubbles carried by the fluid. Each of the bubbles travels through networks based on the local information in its proximity, because the bubble moves by interacting with the carrier fluid around it. We have explicitly used bubbles with sizes slightly larger than the cross-sectional area of the microchannels so that the bubbles interact with the walls of the channels, significantly change the fluidic resistance of the channels they occupy, and affect the flows of the carrier fluid.

In the systems we have developed, single bubbles traversing a network of channels (having the same cross-sectional area everywhere) make binary choices at each junction, and enter the channel possessing the highest volumetric flow rate of the carrier fluid at that junction. Interestingly, when only a single bubble flows through a network, a sequence of such choices does not lead the bubble to follow the shortest or the fastest path from the inlet to outlet. This 
behavior occurs because each of the path-selections by a bubble at a junction is determined only by the relation between the flow rates into the channels connected to that junction.

A system of multiple bubbles simultaneously moving through a network, however, can distribute its fluxes in all channels as a single-phase fluid distributes its flow rates. Bubbles used in this work (with sizes slightly larger than the channel) increase the fluidic resistance of the channel through which they travel. The change of fluidic resistance of one channel affects the flow rates in all channels of the network; hence, when multiple bubbles are introduced into the fluidic network at the same time, they interact with one another by changing the fluidic resistances of the paths they follow. The collective behavior of multiple bubbles approaches that of a single-phase fluid as the resistances added by bubbles become comparable to the original fluidic resistances of channels composing the network.

We intend the systems of bubbles in microfluidic networks to serve as a platform with which to study various problems involving networks. The model system used in this work is similar in some of its aspects to real networks, such as those involving food-webs, data, and microcirculation through the body: the path-selections of components traversing these networks are mostly based on incomplete information that the components experience only locally. 


\section{Experimental}

Sample preparation. We created the microfluidic network and the maze using soft lithography. ${ }^{32-35}$ We designed photomasks with a CAD program and fabricated masters on silicon wafers using photoresist (SU8-25, Microchem). We then silanized the wafers ((tridecafluoro-1,1,2,2-tetrahydrooctyl)trichlorosilane, Gelest) ${ }^{36}$ coated them with polydimethylsiloxane (PDMS, Sylgard) and subsequently cured them to produce the stamps in which the channels were imprinted. We sealed the stamps against glass slides after exposing the slides to oxygen plasma for five minutes and the stamps for one minute. We prefilled networks with a solution comprising 2 wt \% Tween-20 in water to retain the hydrophilic character of the PDMS walls. A syringe pump (Harvard Apparatus) controlled the flow rate of the solutions and a cylinder of $\mathrm{N}_{2}$ (Airgas) with an attached digital pressure gauge delivered the gas. We used a high-speed CCD video camera (Phantom v7) to capture movies of the bubbles traversing the networks.

Bubble generation. In our experimental configurations, a laminar flow of surfactantcontaining water carries the bubbles that are generated at a T-junction (not shown in the figures). ${ }^{37}$ At the pressure of $34.0 \mathrm{kPa}$ and flow rates of $2.4-2.6 \mathrm{~mL} / \mathrm{hr}$ that we used in this work, the capillary number of the bubbles was sufficiently small that it did not break at junctions. The Reynolds number was on the order of $0.1 \sim 1$ in the case of the systems examined in this work, ensuring that the effect of the inertia of any fluidic component (whether in the continuous or dispersed phase) was reduced to a small value. We set the frequency of formation of the bubbles by controlling the pressure applied to the gas stream, and varying the flow rate of the aqueous solution that squeezed off the bubbles. ${ }^{38}$ 
Acknowledgments - This work was supported in part by the U.S. Department of Energy, Office of Basic Energy Sciences, Division of Materials Sciences and Engineering under Award DEFG02-00ER45852. This work was also funded by award number W911NF-09-1-0476 from the Army Research Office, Department of the Army. This work was performed in part at facilities provided by the Center for Nanoscale Systems (CNS), a member of the National

Nanotechnology Infrastructure Network (NNIN), which is supported by the National Science Foundation under NSF award no. ECS-0335765.

\section{References}

1. M. E. J. Newman, SIAM Review, 2003, 45, 167-256.

2. M. Boguna, D. Krioukov and K. C. Claffy, Nat. Phys., 2009, 5, 74-80.

3. $\quad$ R. Albert and A. L. Barabasi, Rev. Mod. Phys., 2002, 74, 47-92.

4. E. N. Dijkstra, Numer. Math., 1959, 1, 269-271.

5. T. H. Cormen, C. E. Leiserson and R. L. Rivest, Introduction to Algorithms, The MIT Press, Cambridge, MA, 1990.

6. O. Steinbock, A. Toth and K. Showalter, Science, 1995, 267, 868-871.

7. T. Nagakaki, H. Yamada and A. Toth, Nature, 2000, 407, 470.

8. K. Agladze, N. Magome, R. Aliev, T. Yamaguchi and K. Yoshikawa, Physica D, 1997 , 106, 247-254.

9. N. G. Rambidi and D. Yakovenchuck, Biosystems, 1999, 51, 67-72.

10. D. R. Reyes, M. M. Ghanem, G. M. Whitesides and A. Manz, Lab Chip, 2002, 2, 113 116.

11. M. J. Fuerstman, P. Deschatelets, R. Kane, A. Schwartz, P. J. A. Kenis, J. M. Deutch and G. M. Whitesides, Langmuir, 2003, 19, 4714-4722.

12. S. R. Hodges, O. E. Jensen and J. M. Rallison, J Fluid Mech., 2004, 501, 279-301.

13. H. Wong, C. J. Radke and S. Morris, J Fluid Mech., 1995, 292, 95-110.

14. F. P. Bretherton, J Fluid Mech., 1961, 10, 166-188.

15. F. Jousse, R. Farr, D. R. Link, M. J. Fuerstman and P. Garstecki, Phys. Rev. E, 2006, 74, 036311-036311-036316.

16. M. J. Fuerstman, P. Garstecki and G. M. Whitesides, Science, 2007, 315, 828-832.

17. M. Schindler and A. Ajdari, Phys. Rev. Lett., 2008, 100, 044501-044501-044504.

18. O. Cybulski and P. Garstecki, Lab Chip, 2010, 10, 484-493.

19. W. Engl, M. Roche, A. Colin, P. Panizza and A. Ajdari, Phys. Rev. Lett., 2005, 95, 208304-208304:4.

20. D. A. Sessoms, M. Belloul, W. Engl, M. Roche, L. Courbin and P. Panizza, Phys. Rev. E, 2009, 80, 016317-016317:10. 
21. J. A. Davis, D. W. Inglis, K. J. Morton, D. A. Lawrence, L. R. Huang, S. Y. Chou, J. C. Sturm and R. H. Austin, Proc. Natl. Acad. Sci. U.S.A., 2006, 103, 14779-14784.

22. O. Cybulski, presented in part at the Coding and Computation in Microfluidics, http://cba.mit.edu/events/07.05.fluid/, 2007.

23. J. Stark and M. Manga, Transp. Porous Med., 2000, 40, 201-218.

24. J. B. Keller, J Fluid Mech., 2003, 480, 61-63.

25. G. K. Batchelor, An Introduction to Fluid Dynamics, Cambridge University Press, 1967.

26. D. R. Link, S. L. Anna, D. A. Weitz and H. A. Stone, Phys. Rev. Lett., 2004, 92, 054503054503:4

27. J. M. Kirshner and S. Katz, Design Theory of Fluidic Components, Academic Press, New York, 1975.

28. The difficulty is due to the fact that the interaction between the bubble and the carrier liquid needs to be integrated around the liquid-gas interface, whose shape itself is determined by the interaction between the bubble and the carrier liquid (i.e., the equation is implicit). In addition, the liquid-gas interface has its own interfacial energy which also affects the movement of a bubble.

29. M. J. Fuerstman, A. Lai, M. E. Thurlow, S. S. Shevkoplyas, H. A. Stone and G. M. Whitesides, Lab Chip, 2007, 7, 1479-1489.

30. S. Xu, Z. Nie, M. Seo, P. Lewis, E. Kumacheva, H. A. Stone, P. Garstecki, D. B. Weibel, I. Gitlin and G. M. Whitesides, Angew. Chem. Int. Ed., 2005, 44, 3799-3799.

31. V. Labrot, M. Schindler, P. Guillot, A. Colin and M. Joanicot, Biomicrofluidics, 2009, 3 , 012804-012820.

32. D. C. Duffy, J. C. McDonald, O. J. A. Schueller and G. M. Whitesides, Anal. Chem., 1998, 70, 4974-4984.

33. J. C. McDonald, D. C. Duffy, J. R. Anderson, D. T. Chiu, H. K. Wu, O. J. A. Schueller and G. M. Whitesides, Electrophoresis, 2000, 21, 27-40.

34. D. Qin, Y. N. Xia and G. M. Whitesides, Adv. Mater., 1996, 8, 917-919.

35. Y. Xia and G. M. Whitesides, Angew. Chem. Int. Ed., 1998, 37, 550.

36. M. K. Chaudhury and G. M. Whitesides, Langmuir, 1991, 7, 1013-1025.

37. T. Thorsen, R. W. Roberts, F. H. Arnold and S. R. Quake, Phys. Rev. Lett., 2001, 86, 4163-4166.

38. P. Garstecki, M. J. Fuerstman, H. A. Stone and G. M. Whitesides, Lab Chip, 2006, 6, 437-446. 

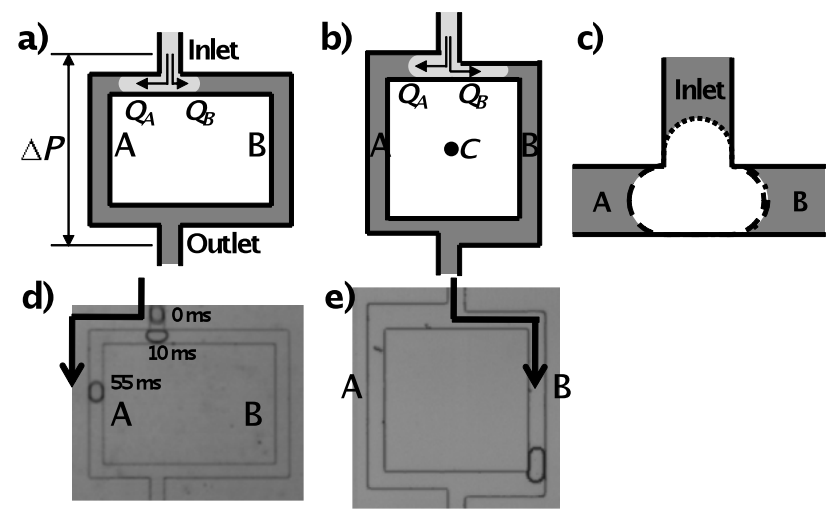

Figure 1. a) A diagram of a single-phase fluid flowing through a branching, two-way intersection. The pressurized $(\Delta P)$ fluid distributes continuously along both paths simultaneously but proceeds at different flow rates, due to the anisotropy of the channel shapes (e.g., channel lengths). b) A diagram of a continuous stream of liquid through a network possessing two branch channels point-symmetric (about point ' $C$ ') to each other. The symmetry of the device guarantees that the fluidic resistances of two paths, and correspondingly the flow rates, are the same $\left(Q_{A}=Q_{B}\right)$. c) A schematic illustrating a bubble at a symmetric T-junction. The movement of the bubble is determined by the interactions between the bubble and the carrier liquid, occurring at three regions at the inlet (dotted line), channel A (dashed line), and channel B (dashdotted line). d) A composite picture of three optical micrographs taken at different moments $(0$, 10, 55 milliseconds) as a single bubble travels a fluidic network. e) The movement of a bubble proceeding through a point-symmetric network similar to the one in b). A single bubble always selects path B. 


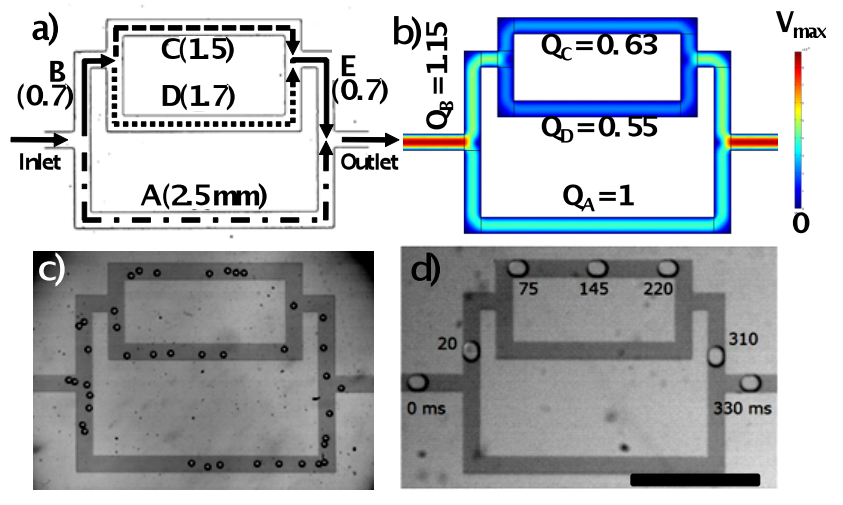

Figure 2. a) A three-path network that comprises five channels, labeled A through E. The numbers in parentheses represent the lengths of the channels ( $\mathrm{mm})$. b) The velocity fields of a Stokes flow through the network, simulated by COMSOL package. The color bar on the right shows the normalized velocity. The relative flow rates through channels $\mathrm{A}, \mathrm{C}$, and D are calculated to be $1,0.63,0.55$. c) The distribution of real fluid flow through the network at a Reynolds number of $\sim 0.25$, obtained by tracking the flow of small bubbles (diameter $\sim 25 \mu \mathrm{m}$; the generation of these bubbles was random) dispersed in the flow. The velocities of particles along channels A, C, and D are 16.6, 9.93, $8.36 \mathrm{~mm} / \mathrm{s}$, forming the ratio of 1: $0.60: 0.50$. d) Composite picture of seven optical micrographs taken as a single bubble travels through the same fluidic network. The numbers indicate the time (ms) at which the bubble reaches a particular location in the network. The scale bar represents $1 \mathrm{~mm}$.
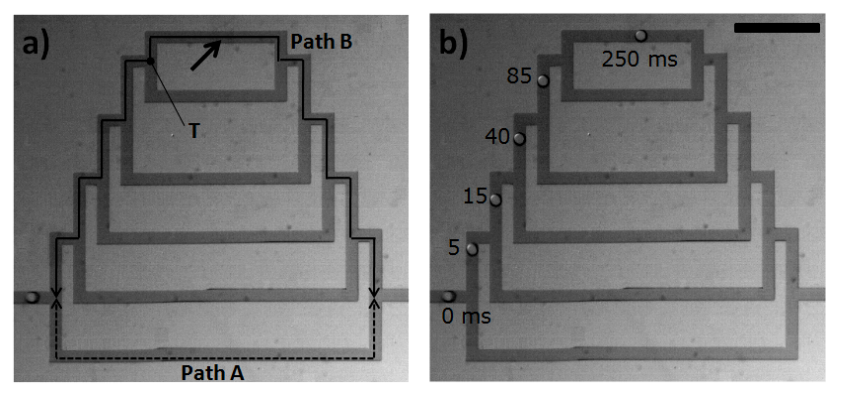

Figure 3. a) A microfluidic network with an upper path that successively bifurcates and merges. $44 \%$ of the injected fluid flows through path A; $4.6 \%$ flows through channel B (indicated with an arrow). b) A composite picture of six optical micrographs taken as a single bubble travels the same fluidic network. The scale bar represents $1 \mathrm{~mm}$. 

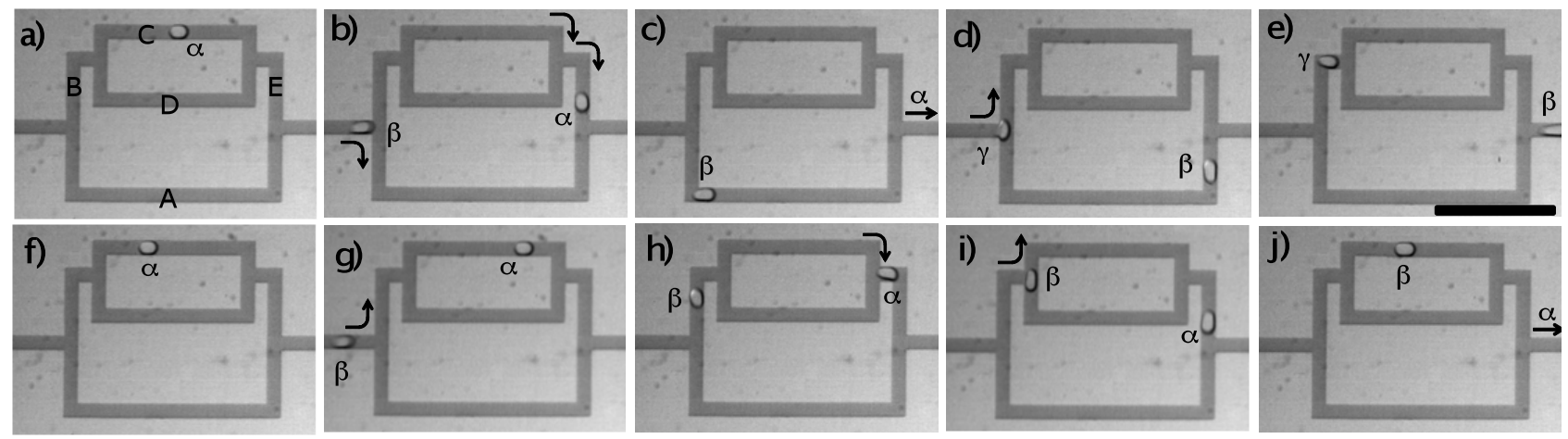

Figure 4. Optical micrographs of multiple bubbles traversing the network simultaneously. a) - e) The pattern of flows at $2.50 \mathrm{~mL} / \mathrm{h}$ flow rate of water. f) $-\mathbf{j}$ ) The pattern of flows at $2.49 \mathrm{~mL} / \mathrm{h}$ flow rate of water. A scale bar in e) represents $1 \mathrm{~mm}$.

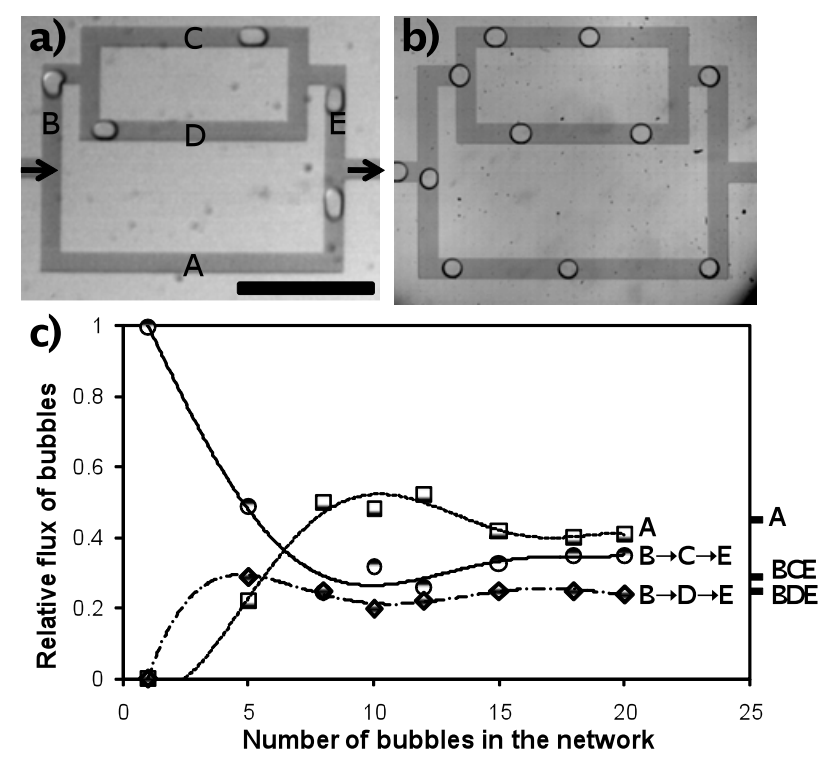

Figure 5. The selection of paths by bubbles, at multiple bubble density levels. The arrow indicates the direction of the flow. a) The selection of paths by bubbles at the density of $\sim 5$ bubbles in the network and b) by bubbles at the density of $\sim 10$ bubbles in the network. c) Relative fluxes (flux in a path over the total flux through the network) of bubbles in three pathways (square, $\mathrm{A}$; circle, $\mathrm{B} \rightarrow \mathrm{C} \rightarrow \mathrm{E}$; diamond, $\mathrm{B} \rightarrow \mathrm{D} \rightarrow \mathrm{E}$ ), plotted versus the number of bubbles in the network. The ticks on the right axis represent the relative, volumetric flow rates of a single-phase fluid without bubbles. Trend lines using $5^{\text {th }}$ order polynomial are drawn to guide the eye. A scale bar in a) represents $1 \mathrm{~mm}$. 